\title{
LAS NIEBLAS EN LA DEPRESION DEL EBRO: EL CASO DEL CAMPO DE ZARAGOZA Y LA HOYA DE HUESCA
}

\author{
M² Luz HERNANDEZ NAVARRO \\ Javier del VALLE MELENDO \\ Universidad de Zaragoza
}

\begin{abstract}
RESUMEN: Este trabajo analiza el fenómeno de las nieblas en dos comarcas del valle del Ebro: el campo de Zaragoza en su sector central y la Hoya de Huesca, en posición más marginal y a mayor altura. Para ello hace, en primer lugar, un estudio estadistico-probabilístico, y termina con un análisis de tipos de tiempo que dan lugar a la aparición de este meteoro, con diferentes matices en sus consecuencias sobre la formación de nieblas en ambas comarcas.
\end{abstract}

\begin{abstract}
This work analyses the fog-activity in two separated districts of the Ebro river basin: The Campo de Zaragoza area, located in its central sector and the Hoya de Huesca area, in a more marginal position and at a higher altitudinal level. Firstly a statistical-probabilistic study is made, followed by an analysis of the types of weather that lead to the apparition of this meteor. ( $M^{2}$. L. Hernández y $J$. del Valle)
\end{abstract}

Sumario: Introducción.- Metodología.- Concepto de niebla.- La niebla en el campo de Zaragoza.- La niebla en la Hoya de Huesca.- Estudio comparativo.- Frecuencia de aparición de nieblas.- Situaciones de tiempo.- Conclusiones.- Bibliografía.

PALABRAS CLAVE: nieblas, Hoya de Huesca, Campo de Zaragoza, frecuencia, situaciones de tiempo.

\section{INTRODUCCION}

El objeto de este estudio es comparar la distribución y frecuencia de las nieblas en dos comarcas aragonesas: el Campo de Zaragoza y la Hoya de Huesca, ambas situadas en la Depresión del Ebro, aunque la segunda con una disposición marginal, próxima ya a las primeras sierras prepirenaicas.

El Campo de Zaragoza es una comarca topográficamente deprimida, ligada estrechamente al Ebro, eje conductor de su evolución geográfica. En su centro se localiza el fondo del Valle, asiento tradicional de los regadíos, que queda enmarcado por cuatro relieves estructurales horizontales de tipo muela: los Montes de Castejón $(744 \mathrm{~m})$ y Sierra de Alcubierre $(812 \mathrm{~m})$ al Norte, y la Muela $(627 \mathrm{~m})$ y La Plana de Zaragoza $(695 \mathrm{~m})$, al Sur. Está recorrido por el río Ebro, al que fluyen el río Gállego, el Huerva y el Jalón, actuando a modo de arterias de agua que vivifican esta región, marcada por la aridez

Esta disposición ejerce sobre el área un efecto de cubeta, cuyo principal efecto es el estancamiento de las masas de aire cuando lo favorece una situación atmosférica estable. Durante el verano sufren un recalentamiento que permite que se alcancen temperaturas muy elevadas. En invierno, se acelera la pérdida de calor nocturna por irradiación y se acumula aire frío en el fondo del valle. con mínimas muy bajas y formación de nieblas por efecto de 
inversión térmica en las capas más bajas. Así, la niebla es persistente en la ribera de los valles fluviales mientras que el cielo está despejado al ascender en altura.

La Hoya de Huesca también constituye, como su nombre indica, una comarca topográficamente deprimida. Es una depresión excavada en el contacto entre los materiales tercierios de la depresión del Ebro y las calizas cretácicas y eocenas del Prepirineo ( Sierras de Guara, Gabardiella, Caballera y Loarre). Así, la comarca queda comprendida entre los relieves residuales de las muelas del Valle del Ebro, al Sur, y los primeros contrefuertes prepirenaicos al Norte, por lo que constituye una zona favorable para la formación de nieblas por irradiación y la acumulación de aire frío en las partes topográficamente más deprimidas. Un rasgo diferenciador con respecto al Campo de Zaragoza es la ausencia de corrientes fluviales importantes, que aporten humedad.

La formación de nieblas está favorecida por situaciones de estabilidad atmosférica ligadas a la presencia de un anticiclón dinámico que se refuerza, en los meses invernales, por efecto térmico. Este enfriamiento facilita la acumulación de aire frío, más denso y pesado, en estas comarcas deprimidas.

Este proceso, que es el esencial para el desarrollo de este meteoro, puede verse ayudado por la presencia de un débil flujo del sureste que aporta humedad y provoca turbulencias internas en la masa de aire.

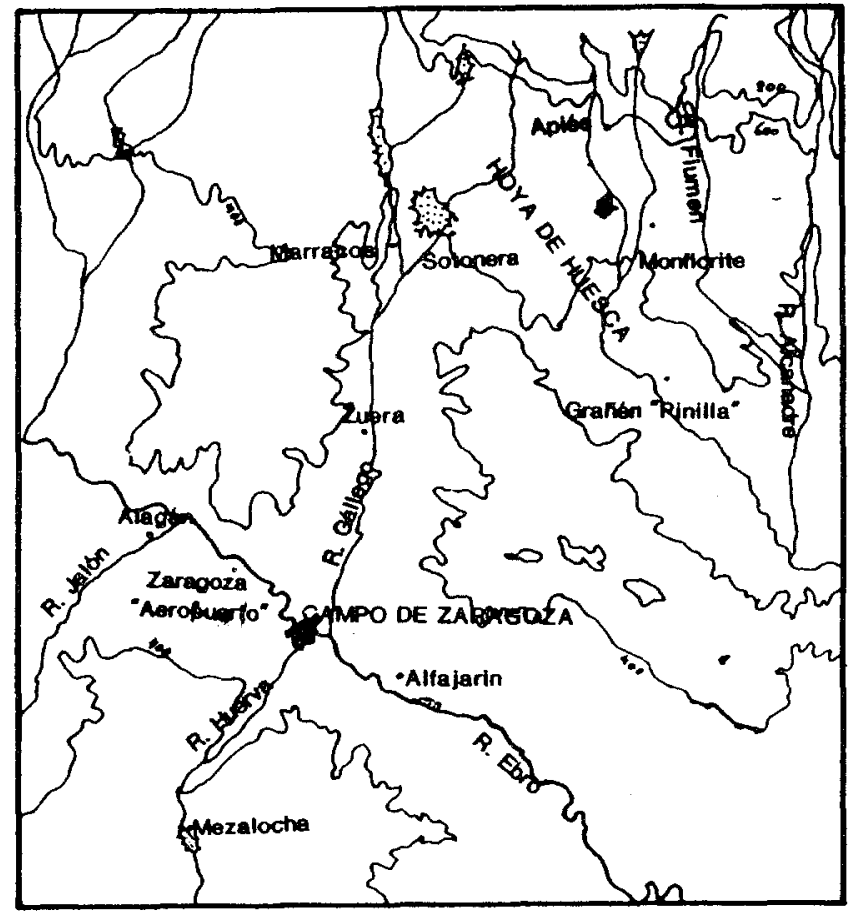

Fig 1.- Esquema topográfico 


\section{METODOLOGIA}

Hemos utilizado las observaciones de este meteoro en las siguientes estaciones meteorológicas del Instituto Nacional de Meteorología:

\author{
ESTACION \\ HOYA DE HUESCA \\ Embalse de la Sotonera \\ Apiés \\ Monflorite \\ Grañén "Pinilla" \\ ESTACION INTERMEDIA \\ Marracos \\ CAMPO DE ZARAGOZA \\ Zaragoza "Aeropuerto" \\ Zuera "El Vedado" \\ Alagón "Gasolinera" \\ Alfajarín \\ Embalse de Mezalocha
}

$\begin{array}{rrrr}\text { ALT. } & \text { LAT.N. } & \text { LONG.O. } & \text { PERIODO } \\ 413 \mathrm{~m} . & 42^{\circ} 06^{\prime} & 0^{\circ} 40^{\prime} & 1955-84 \\ 680 \mathrm{~m} . & 42^{\circ} 13^{\prime} & 0^{\circ} 25^{\prime} & 1970-84 \\ 436 \mathrm{~m} . & 42^{\circ} 06^{\prime} & 0^{\circ} 22^{\prime} & 1955-84 \\ 335 \mathrm{~m} . & 41^{\circ} 57^{\prime} & 0^{\circ} 22^{\prime} & 1955-84 \\ & & & \\ 400 \mathrm{~m} & 42^{\circ} 05^{\prime} & 0^{\circ} 47^{\prime} & 1955-84 \\ & & & \\ 240 \mathrm{~m} . & 41^{\circ} 39^{\prime} & 1^{\circ} 00^{\prime} & 1955-84 \\ 285 \mathrm{~m} . & 41^{\circ} 52^{\prime} & 0^{\circ} 47^{\prime} & 1970-84 \\ 235 \mathrm{~m} . & 41^{\circ} 46^{\prime} & 1^{\circ} 05^{\prime} & 1970-84 \\ 199 \mathrm{~m} . & 41^{\circ} 36 & 0^{\circ} 40^{\prime} & 1970-84 \\ 460 \mathrm{~m} . & 41^{\circ} 25^{\prime} & 1^{\circ} 05^{\prime} & 1955-84\end{array}$

Los observatorios del Embalse de la Sotonera, Monflorite, Zaragoza "Aeropuerto" y Embalse de Mezalocha disponen de 30 años de registro, como recomienda la O.M.M. Del resto de los observatorios hemos tomado el periodo 1970-84 (mínimo común entre ellas) para poder compararlas, aunque en algunos casos dispusieran de series de observación más largas.

En general, se puede afirmar que todas ellas son series continuas y fiables, sin lagunas en los datos, a pesar de ser éste un meteoro del que no siempre se dispone información o, acaso, de forma ocasional.

Hemos tomado los datos de las tarjetas resumen mensuales. A partir de ellas hemos ensayado la comparación entre las dos comarcas, utilizando un tratamiento estadístico que nos ha permitido después hacer un análisis de frecuencias. Esta metodología nos aporta información sobre la validez y homogeneidad de los datos. Nos podemos hacer, de esta forma, una idea de la variación interanual del fenómeno, de la probabilidad de su aparición, y estimar la duración del periodo de retorno de una frecuencia dada, lo que tiene interés para la caracterización climática de estas comarcas y su comparación.

De otra parte, hemos realizado un estudio de situaciones de tiempo que han tenido diferentes consecuencias sobre la formación de nieblas en las dos comarcas de estudio.

\section{CONCEPTO DE NIEBLA}

La niebla se define como una suspensión más o menos densa de gotitas de agua o, en ciertos casos, de cristales de hielo muy finos, que reducen la visibilidad horizontal en las capas de aire en contacto con la superficie del suelo (ANTONIOLETTI et al., 1984). Presenta una gran extensión horizontal y poco espesor y se asocia a situaciones de tiempo estable, frecuentemente anticiclónicas, con inversión térmica. La capa de aire afectada por este proceso cs variable, aunque sucle ser normalmente inferior a los $300 \mathrm{~m}$. 
Es frecuente que las nieblas se formen en la tarde-noche por la pérdida de calor nocturno y que se disipen a mediodía, ya que al estar ligadas a situaciones anticiclónicas, el calentamiento de la masa de aire por la radiación solar permite que el vapor de agua que se contiene en ella permanezca en estado gaseoso. La niebla se vuclve a echar al atardecer.

Según su espesor y densidad se distingue entre bruma, neblina y niebla. Si la visibilidad en horizontal es inferior a $1 \mathrm{~km}$. hablamos de niebla. Es necesario llamar aquí la atención sobre el hecho de que la noción de niebla repose sobre la apreciación de la visibilidad, lo que entraña un cierto número de inconvenientes a la hora del registro y que dificulta enormemente la comparación de los datos proporcionados por distintas estaciones meteorológicas, lo que sólo se podría paliar mediante el uso de aparatos más sofisticados como un captador de la duración de humectación.

Otro inconveniente que se presenta es la consideración como día de niebla aquél en el que el metcoro ha sido observado, independientemente se su intensidad horaria, e incluso la ausencia de su observación durante las horas de la noche, cuando existe la probabilidad más alta de que se produzca el proceso de condensación de vapor de agua en las capas bajas de la atmósfera por efecto del enfriamiento del aire por debajo del punto de rocío.

\section{IA NIEBLA EN EL CAMPO DE ZARAGOZA}

La niebla en uno de los meteoros que más afectan al centro de la Depresión del Ebro, como lo atestiguan los 35,1 días de media anual que se observan en el Aeropuerto de Zaragoza y en el resto de los observatorios situados en el fondo del valle (Alagón: 25,5; Alfajarín: 30,8) o en sus afluentes (Zuera: 25,7). Será necesario alejarse unos kilómetros del fondo del valle y ascender cn altura para ver disminuir su número (Embalse de Mezalocha: 10,3 días) (ver gráfico 1).

La proximidad del Ebro y de otros ejes fluviales como fuentes de humedad aumentan la posiblidad de formación de cstos bancos de nieblas, más frecuentes y persistentes en las cercanías de corrientes de agua. Es el caso de Zaragoza, donde la frecuencia de nieblas es especialmente intensa en la confluencia de los ríos Ebro, Gállego y Huerva. La contaminación urbana e industrial también proporciona abundantes núcleos de condensación. La desembocadura del Gállego es un punto en el que se fijan las nieblas. Unos metros más alto la nicbla ha podido levantarse o ni siquiera haberse formado. Se trata de un fenómeno de inversión lérmica.

El reparto de este metcoro a lo large de los meses guarda estrecha relación con el de la humcdad relativa. Se reparte de septiembre-octubre a marzo, siendo especialmente frecuentc en cl llamado "trimestre de las nieblas" (noviembre,diciembre y enero) registrándose el máximo mensual en diciembre.

Cuadro 1.- Número de días de nicbla en el Campo de Zaragora

$\begin{array}{lcc}\text { ESTACION } & \text { NOV-DIC-EN (dias) \% DEL TOTAL ANUAL } \\ \text { Zaragora "Acropucro" } & 23,7 & 67 \\ \text { Zucra } & 16,5 & 64 \\ \text { Alagón } & 20,9 & 81,9 \\ \text { Alfajarín } & 24,3 & 78,9\end{array}$


$\begin{array}{lll}\text { Mezalocha } \quad 6,9 & 66,9\end{array}$

Fuente: Instituto Nacional de Meteorología

Elaboración propia

Durante el resto del año este fenómeno tiene una escasa incidencia. Ya sólo se registrarán neblinas o acaso brumas en algunos días del verano.

\section{LA NIEBLA EN LA HOYA DE HUESCA}

A pesar de la reducida extensión de la comarca, las diferencias en cuanto a la frecuencia de nieblas entre los diversos observatorios considerados son notables: Apiés registra 9,8 días al año; el Embalse de la Sotonera, 10,4; Monflorite, 23,5 y Grañén "Pinilla" 25,3 (ver gráfica 2).

Los dos observatorios con un mayor número de días de niebla son los situados a menor altura, en amplias llanuras deprimidas, mientras que la frecuencia de este meteoro disminuye notablemente al asceder en altitud. Este hecho nos hace suponer que las nieblas de inversión térmica, cuya formación y persistencia es más fácil en las depresiones y zonas bajas, han de ser una buena parte de las registradas en la comarca.Es muy claro en este sentido el caso de Apiés y Monflorite, dos observatorios muy cercanos en línea recta, sin relieves de entidad entre ellos pero con una importante diferencia en el número de días de niebla registrados $(9,8$ en Apiés y 23,5 en Monflorite, como hemos visto).

La diferencia altitudinal de $240 \mathrm{~m}$. (680 m. de Apiés y $436 \mathrm{~m}$, de Monflorite) nos puede ayudar a explicar este fenómeno y nos hace suponer que frecuentemente el techo de la capa de inversión térmica se sitúa en un nivel intermedio entre las dos estaciones, siendo por lo tanto frecuentes los días en los que Monflorite está cubierto por la niebla, que al mismo tiempo es inexistente en Apiés. Sorprende el escaso número de días de niebla registrados en Sotonera, a pesar de estar situada en una zona deprimida. Suponemos que en este caso influirán factores de subjetividad en la observación, basada, como hemos dicho en criterios de visibilidad.

Los meses en los que la niebla es más frecuente son diciembre en Monflorite, Sotonera y Grañén "Pinilla" y enero en Apiés.

La concentración de los días de niebla es muy clara en los meses de noviembre, diciembre y enero. El porcentaje de días de niebla sobre el total anual de este "trimestre de las nieblas" es muy elevado en todos los observatorios:

Cuadro 2.- Número de días de niebla en la Hoya de Huesca

$\begin{array}{lcc}\text { ESTACION } & \text { NOV-DIC-EN (dias) \% DEL TOTAL ANUAL } \\ \text { Monflorite } & 15,9 & 67,6 \\ \text { Embalsc de Sotonera } & 13,1 & 93,2 \\ \text { Apićs } & 7,2 & 73,4 \\ \text { Grañén "Pinilla" } & 19,9 & 78,6\end{array}$

Fuente: Instituto Nacional de Meteorologia

Elaboración propia 
Durante el resto de los meses del año los días con este meteoro son muy escasos, sólo con cicrta entidad durante los meses de febrero y octubre, incluso no son raros los meses en los que los días de niebla son inexistentes, especialmente durante el periodo estival.

\section{ESTUDIO COMPARATIVO}

Como hemos visto, la niebla es un meteoro más frecuente en el Campo de Zaragoza que en la Hoya de Huesca. La menor altitud de esta comarca, su situación central en el corazón de la Depresión del Ebro y la presencia de importantes corrientes fluviales y áreas de regadío que aportan la humedad necesaria para permitir la condensación, son las razones que explican esta mayor frecuencia. El reparto mensual de nicblas entre ambas comarcas es notablemente similar. Los meses de noviembre, diciembre y enero se conforman como el "trimestre de las nieblas", alcanzando en dicicmbre la máxima frecuencia en casi la totalidad de los observatorios. A lo largo del valle del Gállego encontramos un gradiente que supone una disminución del número de días de niebla a medida que nos alejamos de su desembocadura (Zaragoza "Aeropuerto": 35, 1; Zuera: 25,7; Marracos: 17). Vuelve a aumentar al entrar en la Hoya de Huesca (23,5 en Monflorite; 25,3 en Grañén "Pilnilla").

Entre ambas zonas encontramos el observatorio del Embalse de la Sotonera, que registra, un número sensiblemente inferior de días de niebla. De ser comparables sus registros con los del resto de estaciones consideradas podríamos deducir que esta zona se comports como un umbral que separa la Hoya de Huesca, con un número elevado de días de niebla del Valle del Gállego, que permite la extensión hacia el norte de las nicblas del centro de la Depresión, aunque con una frecuencia progresivamente menor a medida que nos alejamos de él.

\section{FRECUENCIA DE APARICION DE NIEBLAS}

Con objeto de conocer la probabilidad de aparición de nieblas en el Campo de Zaragoza y en la Hoya de Huesca hemos hecho un análisis de frecuencias acumuladas, ordenando los datos en rango creciente y asignándoles a cada uno de ellos una probabilidad. El significado de estos cuadros es el siguiente: en el caso de Zaragoza "Aeropuerto" existe una probabilidad del 1,6\% de que se registren 12 días de nicbla al año o menos, o lo que es lo mismo, una probabilidad del $98.4 \%(100-1,6)$ de que se observen más de 12 días de nicbla en un año.

Por razones de espacio sólo se presentan los resultados anuales de todas las estaciones utilizadas, y los del trimestre de las nicblas en los observatorios más significativos: Monflorite, por la Hoya de Huesca; Marracos, por su situación intermedia y Zaragoza "Acropuceto" del Campo de Zaragora. (cuadros 3,4 y 5 ).

Cuadro 3.- Frecuencia de aparición te nichlas (I)

\begin{tabular}{|ccccccc|}
\hline $\mathrm{i}$ & $\mathrm{F}_{\mathrm{i}}$ & \multicolumn{1}{c}{ E.Solonera } & Monfloric & Marracos & Zaragoza A. Mezalocha \\
\hline 10.016 & 1 & 1 & 6 & 12 & 2 \\
20.050 & 1 & 1 & 7 & 13 & 2 \\
30.083 & 1 & 1 & 7 & 13 & 4 \\
40.117 & 6 & 1 & 9 & 18 & 4 \\
\hline
\end{tabular}




\begin{tabular}{|lrrrrr|}
50.150 & 6 & 2 & 9 & 18 & 4 \\
60.183 & 7 & 5 & 10 & 18 & 4 \\
70.217 & 9 & 9 & 10 & 19 & 5 \\
80.250 & 9 & 10 & 11 & 19 & 5 \\
90.283 & 9 & 11 & 11 & 24 & 6 \\
100.317 & 9 & 13 & 12 & 24 & 6 \\
110.350 & 10 & 15 & 13 & 25 & 6 \\
120.417 & 10 & 16 & 14 & 25 & 6 \\
130.417 & 11 & 17 & 16 & 31 & 6 \\
140.450 & 11 & 19 & 16 & 31 & 7 \\
150.483 & 12 & 23 & 17 & 33 & 7 \\
160.517 & 12 & 23 & 17 & 33 & 8 \\
170.550 & 13 & 30 & 18 & 34 & 8 \\
180.583 & 13 & 32 & 18 & 35 & 9 \\
190.617 & 13 & 32 & 19 & 42 & 9 \\
200.650 & 15 & 32 & 23 & 42 & 10 \\
210.683 & 15 & 33 & 24 & 43 & 10 \\
220.717 & 18 & 42 & 24 & 44 & 11 \\
230.750 & 18 & 43 & 25 & 49 & 11 \\
240.783 & 18 & 44 & 25 & 51 & 11 \\
250.817 & 19 & 44 & 25 & 53 & 13 \\
260.850 & 19 & 46 & 26 & 55 & 13 \\
270.883 & 19 & 48 & 27 & 56 & 15 \\
280.917 & 20 & 48 & 29 & 60 & 21 \\
290.950 & 22 & 50 & 30 & 64 & 42 \\
\hline 300.983 & 26 & 54 & 35 & 69 & 44 \\
\hline Fuente:I.N.M. Elaboración propia & & & \\
\hline
\end{tabular}

Cuadro 4.- Frecuencia de aparición de nieblas (II)

\begin{tabular}{|ccccccc|}
\hline $\mathrm{i}$ & $\mathrm{F}_{\mathrm{i}}$ & Apiés & Grañén & Zuera & Alagón & Alfajarín \\
\hline 10.330 & 2 & 14 & 5 & 18 & 0 \\
\hline 20.100 & 3 & 16 & 14 & 20 & 8 \\
30.166 & 6 & 17 & 16 & 20 & 20 \\
40.233 & 8 & 19 & 16 & 21 & 21 \\
50.300 & 9 & 23 & 17 & 22 & 25 \\
60.366 & 11 & 23 & 17 & 22 & 27 \\
70.433 & 11 & 25 & 20 & 23 & 29 \\
80.500 & 12 & 27 & 21 & 23 & 29 \\
90.566 & 13 & 28 & 21 & 24 & 30 \\
100.633 & 13 & 28 & 22 & 25 & 31 \\
110.700 & 14 & 29 & 25 & 27 & 35 \\
120.766 & 14 & 29 & 26 & 32 & 46 \\
130.833 & 16 & 31 & 26 & 34 & 52 \\
140.900 & 16 & 47 & 29 & 36 & 52 \\
150.966 & 18 & 52 & 32 & 39 & 58 \\
\hline Fucntc:I.N.M. Elaboración propia & \\
\hline \multicolumn{7}{c}{ Elabo } \\
\hline
\end{tabular}


Cuadro 5.- Frecuencia de aparición de nieblas (III) en el "trimestre de las nieblas

\begin{tabular}{|c|c|c|c|}
\hline $\mathrm{F}_{\mathrm{i}}$ & Zaragoza A & Marracos & Monflorite \\
\hline 10.016 & 9 & 3 & 0 \\
\hline 20.050 & 11 & 4 & 0 \\
\hline 30.083 & 11 & 5 & 0 \\
\hline 40.117 & 12 & 5 & 0 \\
\hline 50.150 & 13 & 5 & 0 \\
\hline 60.183 & 15 & 5 & 1 \\
\hline 70.217 & 15 & 6 & 6 \\
\hline 80.250 & 16 & 7 & 6 \\
\hline 90.283 & 17 & 7 & 7 \\
\hline 100.317 & 18 & 7 & 8 \\
\hline 110.350 & 18 & 7 & 9 \\
\hline 120.417 & 18 & 9 & 9 \\
\hline 130.417 & 19 & 10 & 10 \\
\hline 140.450 & 21 & 10 & 12 \\
\hline 150.483 & 22 & 11 & 14 \\
\hline 160.517 & 22 & 11 & 18 \\
\hline 170.550 & 25 & 11 & 18 \\
\hline 180.583 & 27 & 11 & 19 \\
\hline 190.617 & 29 & 13 & 20 \\
\hline 200.650 & 29 & 16 & 21 \\
\hline 210.683 & 30 & 17 & 21 \\
\hline 220.717 & 31 & 17 & 21 \\
\hline 230.750 & 31 & 18 & 24 \\
\hline 240.783 & 31 & 19 & 26 \\
\hline 250.817 & 34 & 22 & 26 \\
\hline 260.850 & 36 & 22 & 26 \\
\hline 270.883 & 36 & 23 & 28 \\
\hline 280.917 & 37 & 23 & 30 \\
\hline 290.950 & 38 & 23 & 34 \\
\hline 300.983 & 41 & 25 & 35 \\
\hline
\end{tabular}

\section{SITUACIONES DE TIEMPO}

Como hemos comentado, las situaciones más favorables para la presencia de nicblas en el Valle del Ebro son aquéllas en las que durante los meses invernales se sitúa un anticiclón dinámico, reforzado en superficie por efecto térmico. Suelen ser situaciones muy estables, que sc prolongan durante varios días y que generan inversiones térmicas.

Mucho menos abundantes son las de tropo advectivo, en las que llega un ligero flujo de Sureste, más cálido y húmcdo, que es susceptible de producir nicblas.

Vamos a analizar tres situacioncs del primer tipo

I 24 noviembre 1973

Un núclco de altas presiones, con un amplio centro, de $1028 \mathrm{mb}$. ocupa cl Mediterránco nccidental. Francia y su costa occidental y todo el cuadrante noreste de la Península Ibérica. Las 
borrascas quedan muy alejadas, una de ellas en el norte de Escocia y otra al oeste de las Azores. En altura la estabilidad es absoluta, con $-16^{\circ}$ a $\operatorname{los} 5.500 \mathrm{~m}$. sobre la vertical de la Península.

Esta situación produjo nieblas generalizadas en el Valle del Ebro y en el norte de Cataluña, que no levantaron durante todo el día. El registro de horas de sol en ese día del observatorio en Zaragoza "Aeropuerto" y de Monflorite fue de 0 horas.

La situación se prolongó durante dos días más y termina con la entrada de aire del Norte que disipa las nieblas.

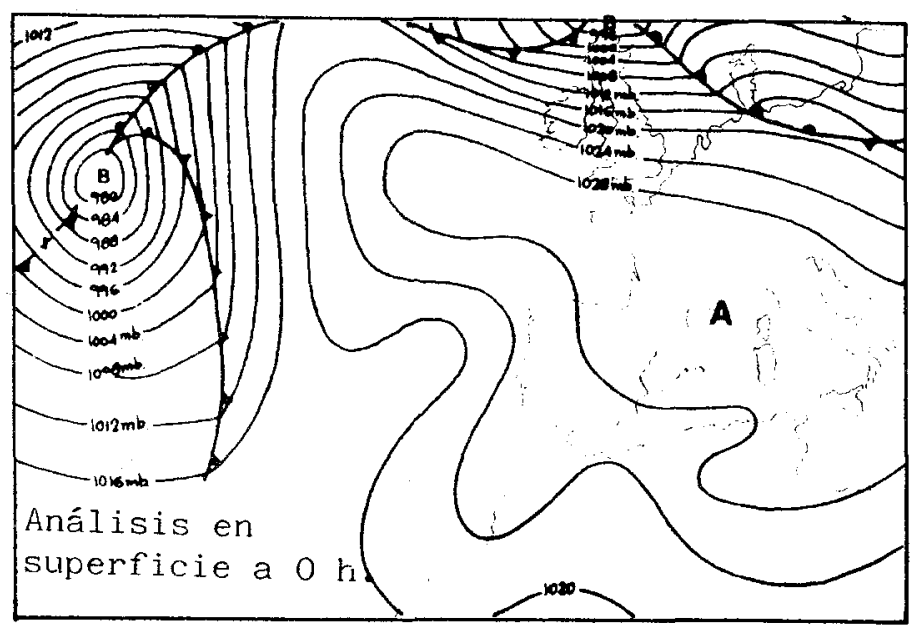

\section{1 enero 1974}

Un potente Anticiclón, con $1032 \mathrm{mb}$. de presión máxima, centrado sobre los Paises Bajos afecta al norte y noroeste de la Península. Otro centro de $1024 \mathrm{mb}$. se localiza en Marruecos, y entre ellos un puente anticiclónico sobre el resto de la Península, excepto en las costas mediterráneas que se ven influidas por una borrasca relativa centrada en Cerdeña (1016 mb.).

En altura, esta borrasca mediterránea se corresponde con un embolsamiento de aire frío ($\left.28^{\circ}\right)$ sin repercusión peninsular. En el resto, la situación es de total estabilidad.

La consecuencia de esta situación es la presencia de nieblas persistentes sobre la Hoya de Huesca (0 horas de sol en Monflorite).

Este meteoro no se registró en el Aeropuerto de Zaragoza (2,8 horas de sol). El débil gradiente de presión entre el Anticiclón europeo y la Borrasca mediterránea provocó un ligero viento del noroeste (cierzo) en el centro del valle que impidió la formación de nieblas sobre Zaragoza. 


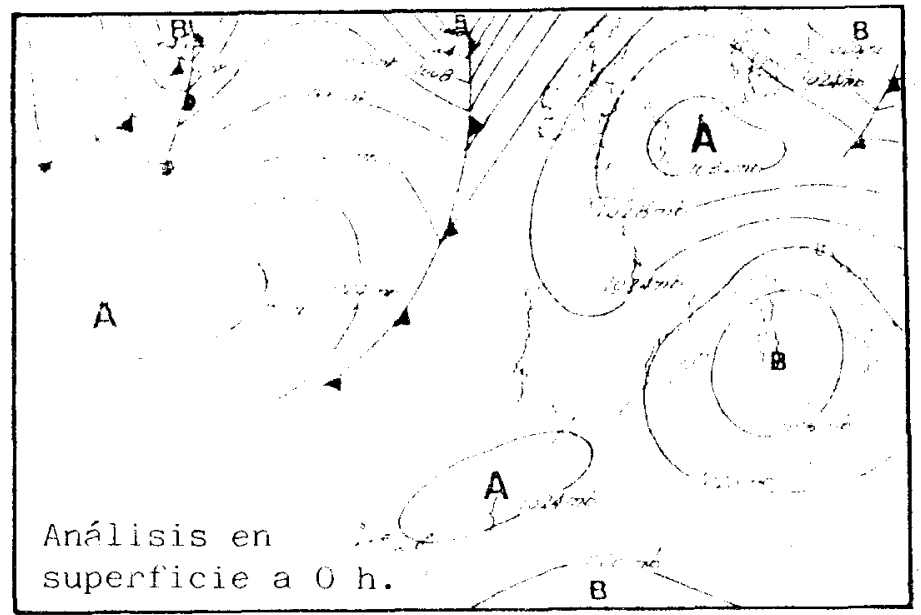

\section{21 noviembre 1973}

En superfice encontramos un área de alta presión $(1028 \mathrm{mb}$.), centro secundario de un amplio anticicón que afecta a toda Europa occidental, Maditerráneo occidental, Islas Británicas y España, con las máximas presiones sobre los Países Bajos y Baviera (1036 mb.). La estabilidad de superficie se corresponde con las capas altas de la atmósfera $\left(-16^{\circ}\right.$ a $5.000 \mathrm{~m}$. $)$.

La consecuencia de esta situación fue niebla persistente en Zaragoza, que sólo registó media hora de sol y de niebla mucho menos intensa en Huesca, que disfrutó de más de 7 horas de sol, observándose este metcoro sólo en la tarde y noche.

Es un ejemplo típico de situación favorable a las nieblas pero con más intensidad de Zaragoza que en Huesca, reflejo de la mayor facilidad para la formación de este fenómeno en tierras ribereñas con respecto a las más marginales.

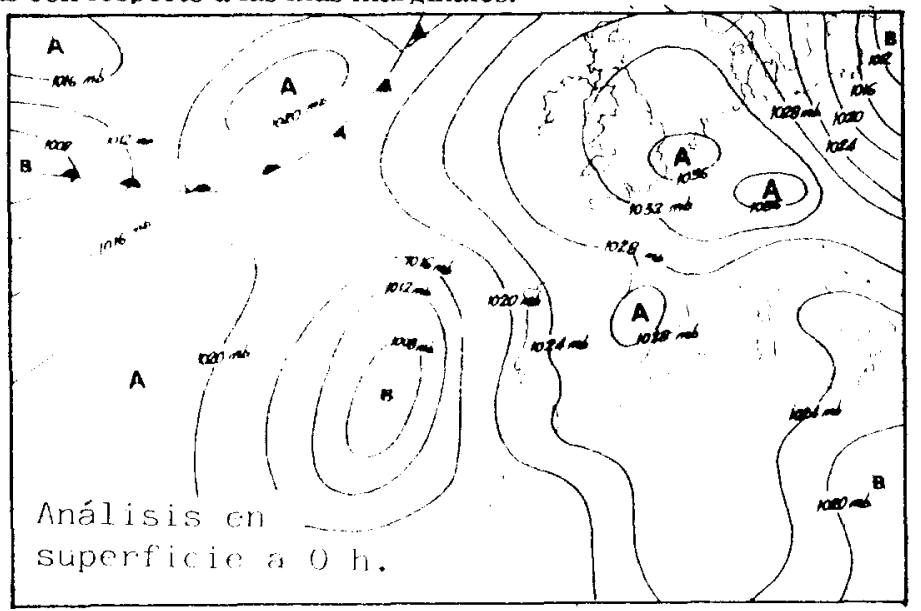




\section{CONCLUSIONES}

1.-La niebla es un fenómeno generalizado en las dos comarcas estudiadas: el Campo de Zaragoza y la Hoya de Huesca, especialmente intenso en los meses de noviembre, diciembre y enero, el "trimestre de las nieblas".

2.-La formación de nieblas de asocia, en general, a tipos de tiempo estables, ligadas a la presencia de un anticiclón, en cuyo centro el escaso gradiente barométrico y la consiguiente ausencia de vientos favorece especialmente la aparición de este meteoro.

3.-La frecuencia de aparición y persistencia de las nieblas es mayor en el Campo de Zaragoza que en la Hoya de Huesca. A esta diferencia contribuye la menor altitud topográfica y la posición central dentro de la cuenca de la comarca zaragozana con respecto a la Hoya de Huesca y la mayor presencia de fuentes de humedad-corrientes fluviales y regadíos principalmente-.

4.-El número de días de niebla va disminuyendo a medida que nos alejamos de la zona central de la Depresión del Ebro. Este gradiente lo podemos observar claramente en las estaciones situadas a lo largo del Valle del río Gállego, que tienen un registro de este meteoro progresivamente menor a medida que ascendemos hacia el norte. Su frecuencia vuelve a aumentar en la Hoya de Huesca por la disposición en cubeta de esta comarca, aunque sin alcanzar los valores del Campo de Zaragoza, por su posición marginal dentro del valle.

\section{BIBLIOGRAFIA}

-ANTONIOLETTI, R.; PINGUET, A.; RIPOCHE-WATCHER, D. (1984). Etude de la frequence des brumes et brouillards dans la region de Chateauneuf-du-Pape (Site des Armeniers) sur la periode 1953-1982. I.N.R.A. s.t.e.f.c.e. Note interne M/84/3. Avignon. 36 p.

-HERNANDEZ NAVARRO, M.L. (en prensa). Agroclimatología de Zaragoza. Institución "Fernando el Católico". Diputación Provincial de Zaragoza.

-VALLE MELENDO, J. del (1987). El clima de la Hoya de Huesca. Departamento de Geografía y y Ordenación del Territorio. Universidad de Zaragoza. Tesis de licenciatura (inédita).

V.V.A.A. (1984). Geografta de Aragón. Tomo I. Ed. Guara. Zaragoza.
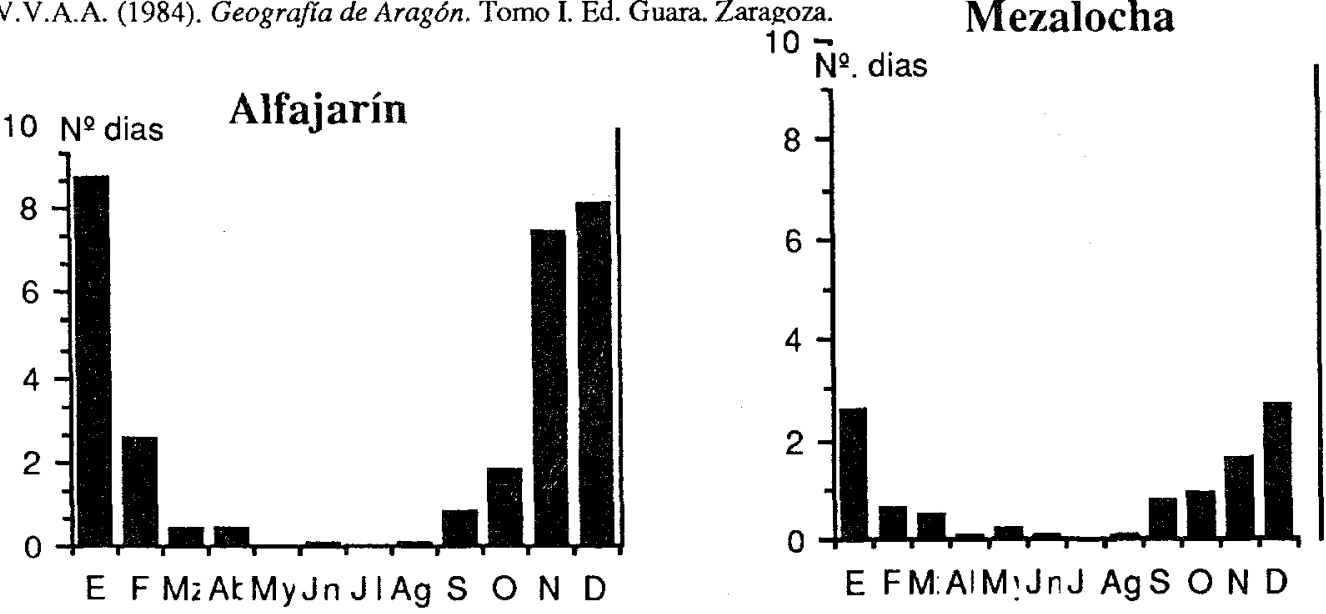

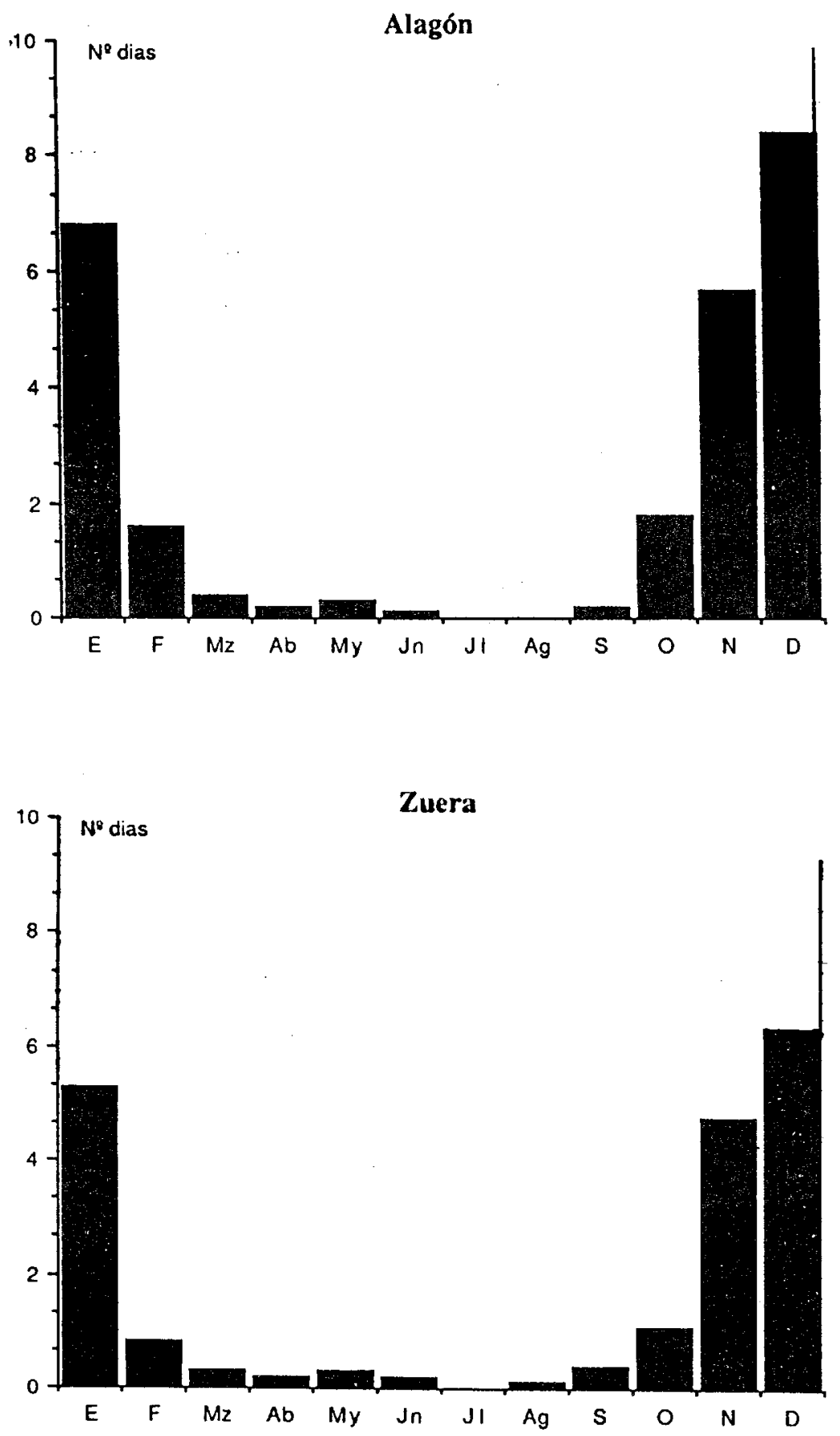

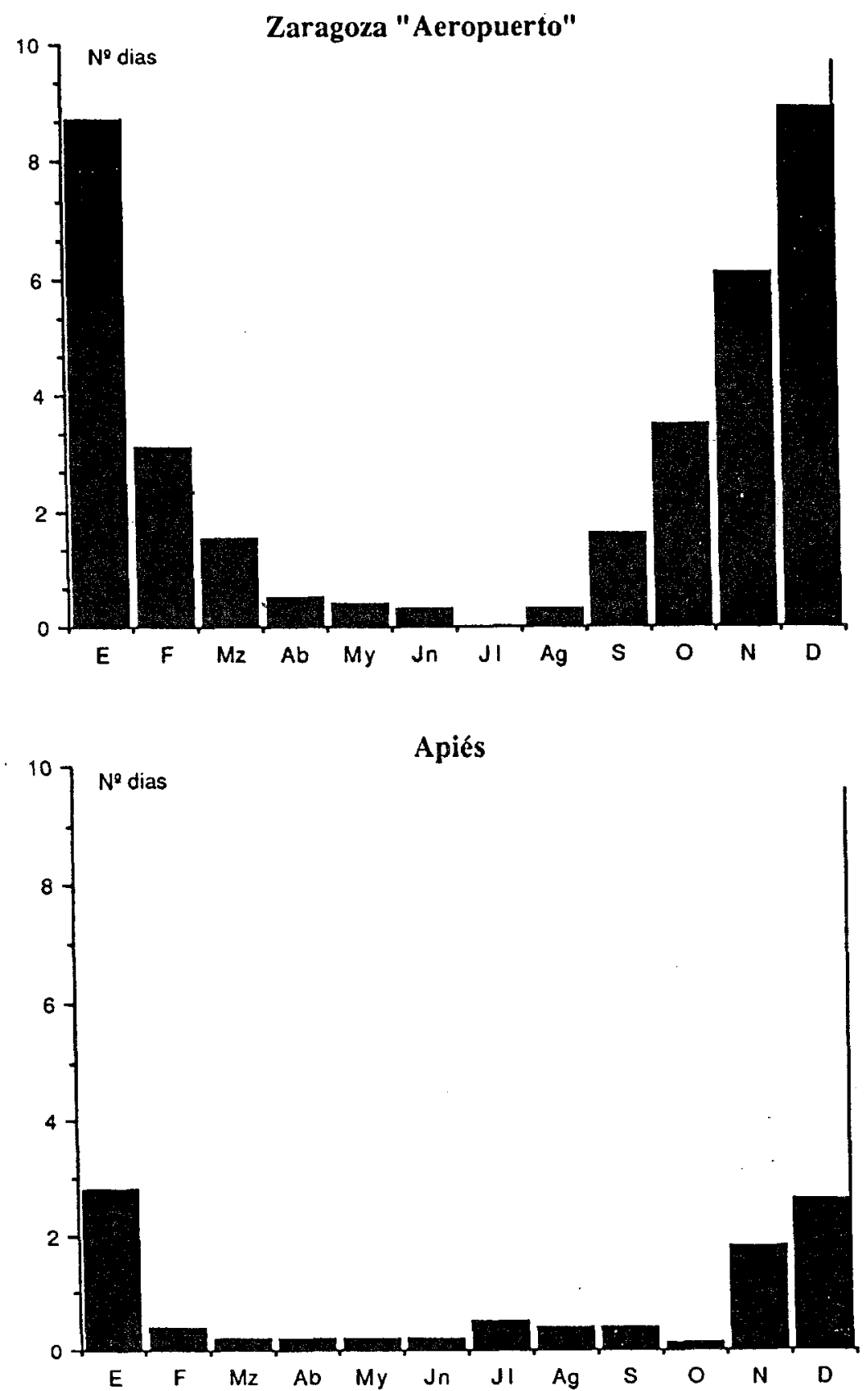

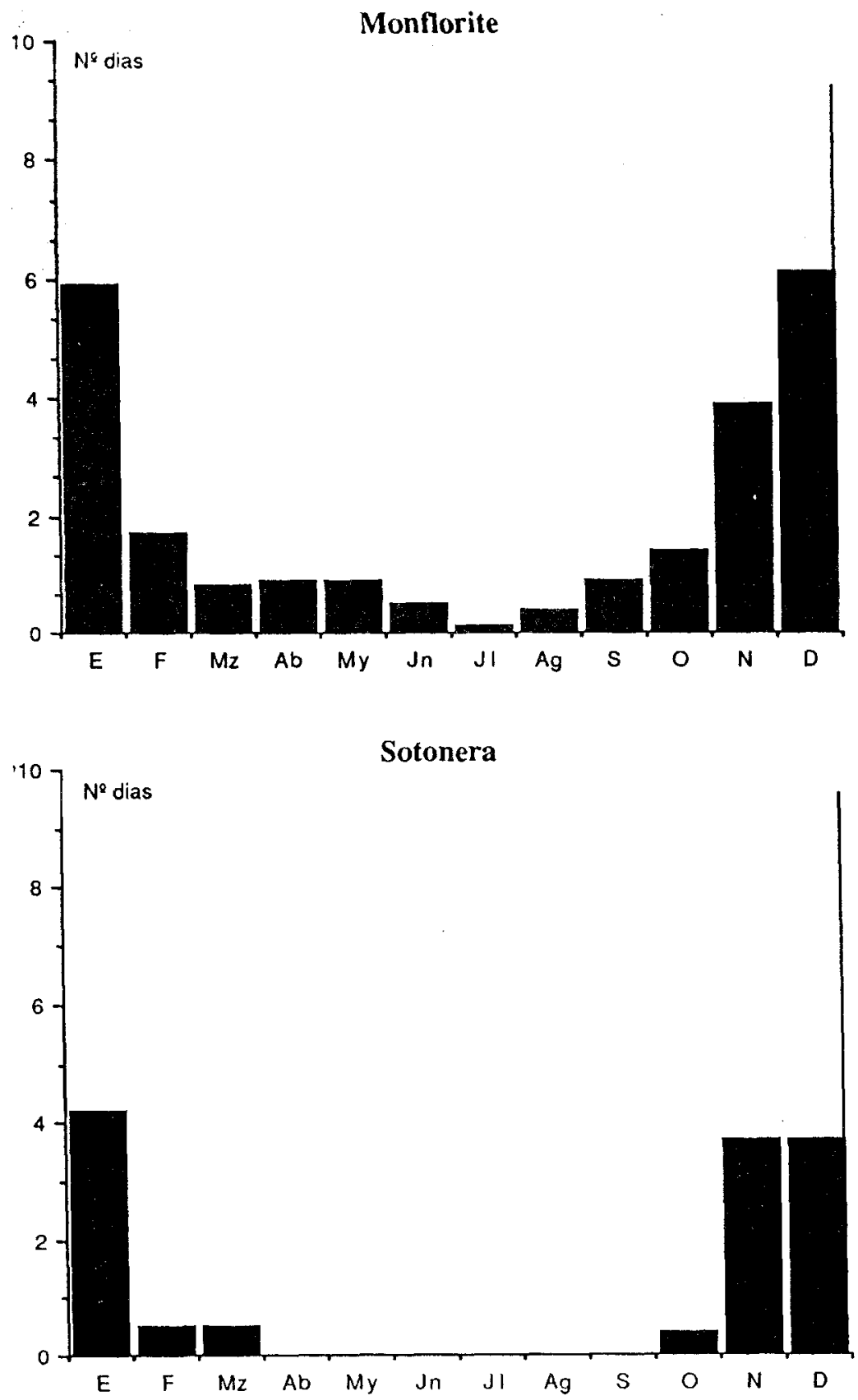

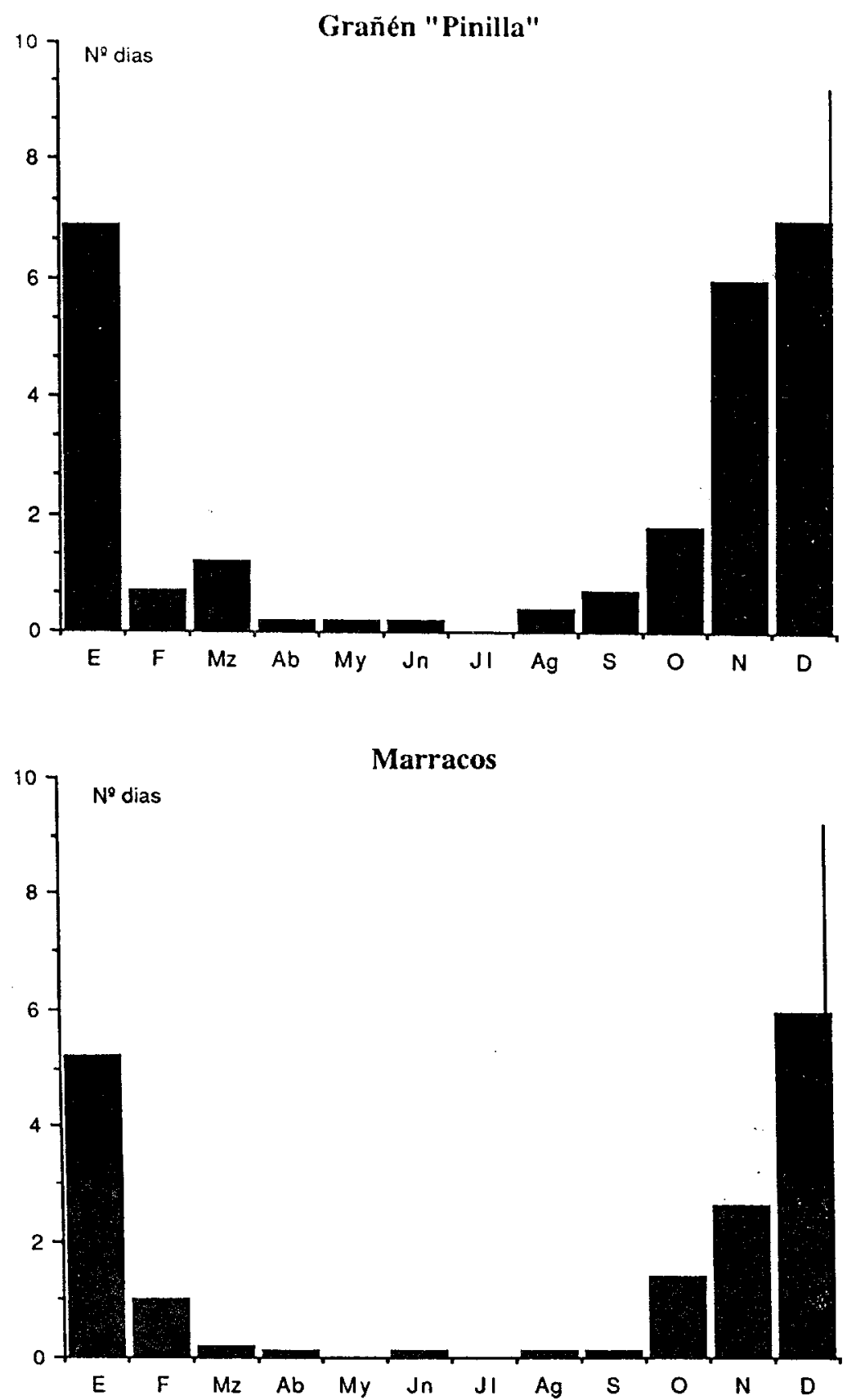